\title{
Greater Omentum
}

National Cancer Institute

\section{Source}

National Cancer Institute. Greater Omentum. NCI Thesaurus. Code C32697.

A large fold of peritoneum that hangs down from the stomach, and extends from the stomach to the posterior abdominal wall after associating with the transverse colon. 